\title{
Parton Distributions from Light-front Holographic QCD
}

\section{Tianbo Liu*}

Thomas Jefferson National Accelerator Facility, Newport News, VA 23606, USA

E-mail: liutbejlab.org

\begin{abstract}
We present a new approach to parton distributions based on light-front holography and Veneziano duality. In this framework, parton distributions can be determined up to a universal reparametrization function $w(x)$, which is highly constrained by boundary conditions. The Regge behavior at small $x$ and the perturbative QCD counting rule at large $x$ are reproduced. Furthermore, unpolarized and polarized distributions are strictly related by the chirality separation from the holographic Lagrangian. It allows us to predict polarized distributions from unpolarized distributions without adding free parameters. The numerical results agree with existing experimental data and the perturbative QCD helicity retention prediction at $x \rightarrow 1$ limit, and particularly, the down quark polarized distribution is predicted to change sign around $x \sim 0.8$. This predictions bears minimal theoretical uncertainty and can be tested soon in future JLab experiments.
\end{abstract}

Light Cone 2019 - QCD on the light cone: from hadrons to heavy ions - LC2019

16-20 September 2019

Ecole Polytechnique, Palaiseau, France

\footnotetext{
${ }^{*}$ Speaker.
} 


\section{Introduction}

In this talk, we present a novel approach $[1,2,3]$ to calculate unpolarized and polarized PDFs based on LFHQCD and the Veneziano duality [4]. This approach allows a determination of (generalized) PDFs from form factors up to a universal reparametrization function $w(x)$, which is highly constrained by boundary conditions. The Regge behavior at small $x$ and the perturbative QCD (pQCD) inclusive counting rule at large $x$ are both reproduced analytically. Taking a simple ansatz of the $w(x)$ satisfying all boundary conditions, one can precisely describe PDFs with minimal number of parameters. The predictive power is kept by the universality of the $w(x)$ for different flavors and different hadrons. Furthermore, the unpolarized and polarized PDFs are strictly related in this approach, which allows us to predict polarized PDFs from unpolarized PDFs without adding free parameters. This property also serves as a test of our framework. With all parameters determined from the unpolarized case, our prediction of polarized PDFs agrees with the experimental data and the $\mathrm{pQCD}$ helicity retention constraint. In particular, the down quark polarized distribution is predicted to have a sign change, which has not been indicated by existing data, around $x \sim 0.8$. It will be tested soon in future experiments.

\section{Unpolarized parton distributions}

Hadrons as the eigenstates of QCD light-front Hamiltonian, one can expand a hadron state on a complete Fock state basis in terms of quarks and gluons degrees of freedom quantized at fixed light-front time. We consider a twist- $\tau$ state, a component with effectively $\tau$ constituents, in the expansion of the nucleon state. The holographic expression of the Dirac form factor $F_{1}(t)$ is

$$
F_{1, \tau}(t)=c_{V, \tau} F_{V, \tau}(t)+c_{V, \tau+1} F_{V, \tau}(t),
$$

with

$$
F_{V, \tau}(t)=\frac{1}{N_{V, \tau}} B\left(\tau-1, \frac{1}{2}-\frac{t}{4 \lambda}\right),
$$

where $t=-Q^{2}$ is the square of the transferred momentum. The subscript $V$ indicates the coupling to a vector current. The $c_{V, \tau}$ and $c_{V, \tau+1}$ are coefficients to be determined and $N_{V, \tau}$ is a normalization factor. The universal mass scale parameter $\lambda$ is fixed by hadron spectroscopy. The two terms in Eq. (2.1) correspond to the contribution the two chiral components, $\Psi_{+}$and $\Psi_{-}$, of the bulk field solution, respectively [5]. For each term, it has the same structure as a generalization of the Veneziano amplitude $B(1-\alpha(s), 1-\alpha(t))$ [4] to non-strong process [6, 7] with the $s$-dependence replaced by a constant, which determines the asymptotic behavior, $(1 / t)^{\tau-1}$, of the form factor for large negative values of $t[6,7]$. Our framework thus incorporates nonperturbative analytic structures found in pre-QCD studies. The $t$-dependence is given by

$$
\alpha_{V}(t)=\frac{1}{2}+\frac{t}{4 \lambda}
$$

which is just the $\rho / \omega$ trajectory in LFHQCD for massless quarks. The quark mass correction is obtained by a shift of the intercept [2]. Such correction is negligible for $u$ and $d$ quarks but essential for $s$ quarks. 
With the Euler integral of the Beta function, we can express the generalized PDF $H(x, \xi, t)$ at zero skewness as

$$
H_{\tau}(x, \xi=0, t)=q_{\tau}(x) \exp [t f(x)]
$$

Here the unpolarized PDF $q_{\tau}(x)$ and the profile function $f(x)$ are given by

$$
\begin{aligned}
q_{\tau}(x) & =\frac{1}{N_{V, \tau}} w(x)^{-\frac{1}{2}}[1-w(x)]^{\tau-2} w^{\prime}(x), \\
f(x) & =\frac{1}{4 \lambda} \log \left(\frac{1}{w(x)}\right)
\end{aligned}
$$

where $w(x)$ is a universal reparametrization function, satisfying the boundary conditions

$$
\begin{aligned}
& w(0)=0, \quad w(1)=1, \quad w^{\prime}(x)>0 \text { for } x \in[0,1), \\
& w^{\prime}(1)=0, \quad w^{\prime \prime}(1)<0 .
\end{aligned}
$$

Then for a twist- $\tau$ state, the unpolarized PDF is given by

$$
q(x)=c_{V, \tau} q_{\tau}(x)+c_{V, \tau+1} q_{\tau+1}(x),
$$

which has the small- $x$ Regge behavior $q(x) \sim x^{-\frac{1}{2}}$ and the large- $x$ pQCD inclusive counting rule $q(x) \sim(1-x)^{2 \tau-3}$.

For numerical results, we need a truncation of the Fock state expansion. Here we consider three cases: (i) only the valence state; (ii) including states with a $u \bar{u}$ or $d \bar{d}$ pair; (iii) also including the state with an $s \bar{s}$ pair. The expansion coefficients $c_{V, \tau}$ are fixed by matching experimentally extracted electromagnetic form factors. Taking a simple form of the $w(x)$ as in Ref. [1], we have the unpolarized PDFs in agreement with global fits, as shown in Fig. 1. We note that one can in principle any other functional form of $w(x)$ as long as all boundary conditions are satisfied. The predictive power is from the universality of $w(x)$ for all parton flavors as well as for different hadrons.

\section{Polarized parton distributions}

To calculate the polarized PDF, one needs the coupling of an axial current rather than a vector current in the unpolarized case. As the current operator only differs by a $\gamma_{5}$, the axial form factor $F_{A, \tau}(t)$ has the same structure as $F_{V, \tau}(t)$ but a sign-flip for the second term, which is the one from the chiral-odd component. Thus, we can express the axial form factor for a twist- $\tau$ state as

$$
F_{A}(t)=c_{A, \tau} F_{A, \tau}(t)-c_{A, \tau+1} F_{A, \tau+1}(t)
$$

where

$$
F_{A, \tau}(t)=\frac{1}{N_{A, \tau}} B\left(\tau-1,1-\frac{t}{4 \lambda}\right)
$$




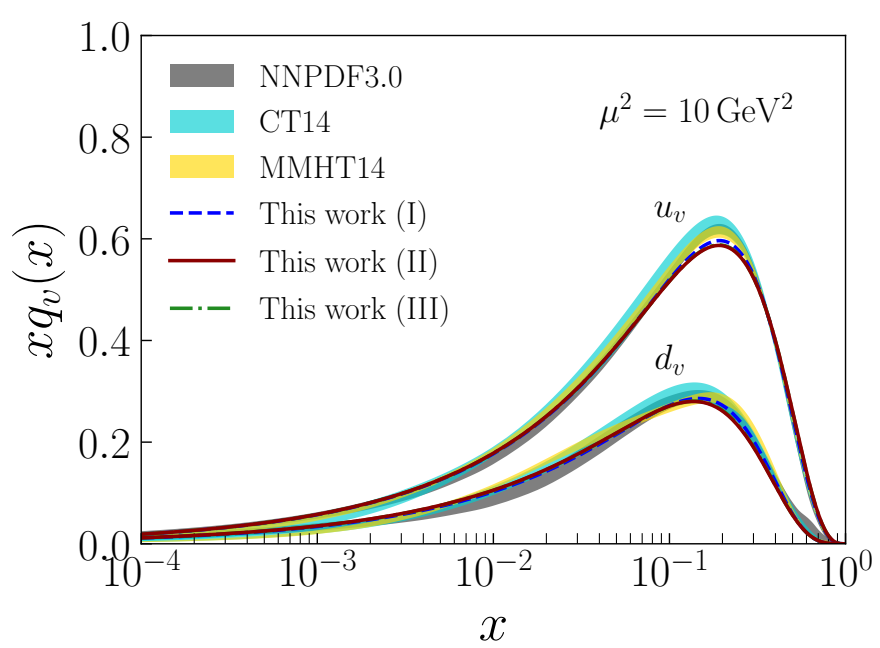

Figure 1: Valence quark distributions in comparison with global analyses. Our results are evolved from the initial scale $\mu_{0}=1.06 \mathrm{GeV}$, which is the matching scale determined from the study of the strong coupling constant [8].

where the subscript $A$ indicates the coupling to an axial current. The $t$-dependence is given by the Regge trajectory of axial mesons,

$$
\alpha_{A}(t)=\frac{t}{4 \lambda}
$$

Because the form factors $F_{A, \tau}(t)$ and $F_{V, \tau}(t)$ are evaluated from the same state, the coefficients $c_{A, \tau}$ and $c_{V, \tau}$ are strictly related by

$$
\frac{c_{V, \tau}}{N_{V, \tau}}=\frac{c_{A, \tau}}{N_{A, \tau}}
$$

Since the normalization convention is arbitrary, one can set $N_{V, \tau}=N_{A, \tau}=N_{\tau}$ for simplicity and identify the coefficients as $c_{V, \tau}=c_{A, \tau}=c_{\tau}$.

The polarized PDF for a twist- $\tau$ state is then given by

$$
\Delta q(x)=c_{\tau} \Delta q_{\tau}(x)-c_{\tau+1} \Delta q_{\tau+1}(x),
$$

where

$$
\Delta q_{\tau}(x)=\frac{1}{N_{\tau}}[1-w(x)]^{\tau-2} w^{\prime}(x) .
$$

The function $w(x)$ is the same as the one in the unpolarized PDF. Therefore, once the coefficients $c_{\tau}$ and the $w(x)$ is determined from unpolarized PDFs, we can predict polarized PDFs without adding free parameters.

With linear combinations of unpolarized and polarized distributions, one can define the spinaligned and spin-antialigned distributions as

$$
q_{\uparrow}(x)=\frac{1}{2}[q(x)+\Delta q(x)], \quad q_{\downarrow}(x)=\frac{1}{2}[q(x)-\Delta q(x)] .
$$


At large- $x$ limit, we find

$$
q_{\uparrow}(x) \rightarrow c_{\tau} q_{\tau}(x), \quad q_{\downarrow}(x) \rightarrow c_{\tau+1} q_{\tau+1}(x) .
$$

The spin-aligned or spin-antialigned distributions tend respectively to a pure contribution from the chiral-even or chiral-odd component of the nucleon bulk field solution. Both of them satisfy the pQCD inclusive counting rule [9, 10,11]. For a valence quark, dominated by $\tau=3$ state at large $x$, the spin-aligned distribution falls off as $(1-x)^{3}$ and the spin-antialigned distribution falls off as $(1-x)^{5}$. Higher order quantum corrections will introduce logarithmic factors but leave the power behavior unchanged.

As the spin-antialigned distribution decreases faster when $x$ goes to 1, the helicity asymmetry $\Delta q(x) / q(x)$ achieves 1 in the $x \rightarrow 1$ limit, consistent with pQCD helicity retention at large $x$. At small $x, \Delta q(x)$ decreases faster than $q(x)$ and the helicity asymmetry drops as $x^{\frac{1}{2}}$. When $x \rightarrow 0$, the helicity asymmetry goes to zero, which indicates that the helicity correlation between a quark and its parent nucleon disappears. This result is a natural expectation [10], because the constituents and the nucleon have infinite relative rapidity for $x \sim 0$.

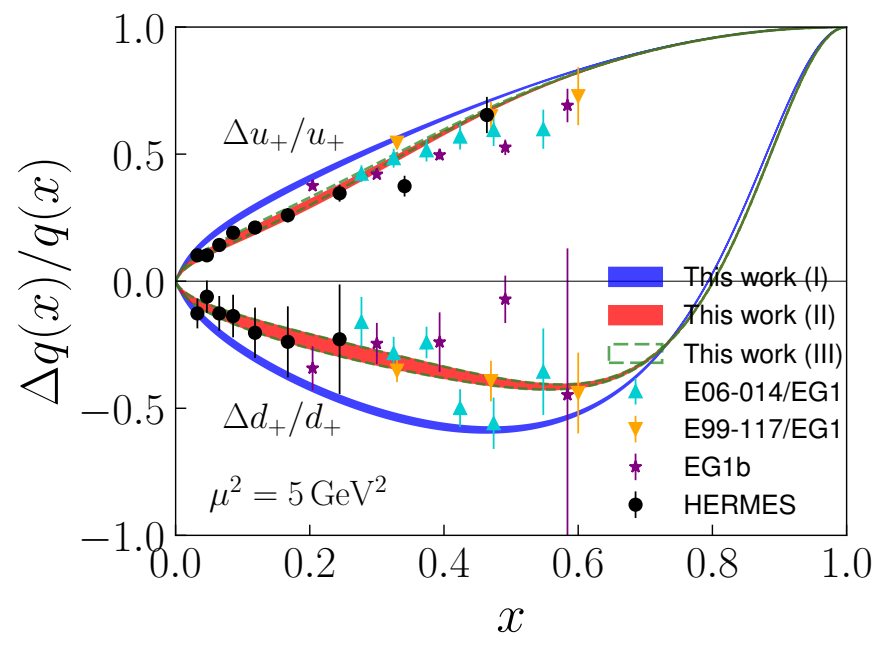

Figure 2: Helicity asymmetries of $u+\bar{u}$ and $d+\bar{d}$ compared with measurements.

For numerical results, we take the same coefficients $c_{\tau}$ and $w(x)$ in the calculation of unpolarized PDFs. As the electromagnetic form factors only measure the difference between quark and antiquark contributions, namely $c_{\tau, u}=u_{\tau}-\bar{u}_{\tau}, u_{\tau}$ and $\bar{u}_{\tau}$ are uniquely separated by the electromagnetic form factors. One needs other observables that are sensitive to quark and antiquark contributions individually to fully separate them. Instead of a full flavor separation, we here use the relation of the isovector axial charge,

$$
g_{A}=(\Delta u+\Delta \bar{u})-(\Delta d+\Delta \bar{d}),
$$

to constraint the sea quark distributions. By doing this, we shift $u_{\tau} \rightarrow u_{\tau}+\delta_{\tau, u}, \bar{u}_{\tau} \rightarrow \bar{u}_{\tau}+\delta_{\tau, u}$ and similarly for the $d$-quark to saturate $g_{A}$. The variation from different ways of such shift is taken as part of our theoretical uncertainty. The results of helicity asymmetries in comparison 
with experimental data are shown in Fig. 2. Our results are in agreement with existing data in the experimentally covered region. In particular, a sign change of the down quark distribution is predicted around $x \sim 0.8$. This prediction bears minimal theoretical uncertainty, because the large- $x$ regime is dominated by the valence state whose coefficients $c_{\tau}$ are well constrained by electromagnetic form factors. It can be tested in future experiments at JLab [12, 13].

\section{Summary}

We present a novel approach to calculate PDFs in the framework of LFHQCD. The PDFs, as well as GPDs, can be determined up to a universal function $w(x)$, which is highly constrained by several boundary conditions. Regge behavior at small $x$ and pQCD constraints at large $x$ are reproduced. Unpolarized and polarized PDFs are strictly related by the chirality separation, which allows a prediction of polarized PDFs from unpolarized PDFs or vice verse, and motivates a simultaneous extraction of unpolarized and polarized PDFs including helicity retention at large $x$. With parameters determined from unpolarized distributions and electromagnetic form factors, our prediction of polarized distributions agree with the experimental data. In addition, a sign change, which is not indicated by existing data because of the limited kinematic coverage, of the down quark polarized distribution is predicted around $x \sim 0.8$. It will be tested soon in future experiments.

\section{Acknowledgments}

This work is supported in part by the U.S. Department of Energy, Office of Science, Office of Nuclear Physics under contract DE-AC05-06OR23177.

\section{References}

[1] G. F. de Teramond et al., Phys. Rev. Lett. 120, no. 18, 182001 (2018).

[2] R. S. Sufian et al., Phys. Rev. D 98, no. 11, 114004 (2018).

[3] T. Liu et al., arXiv:1909.13818 [hep-ph].

[4] G. Veneziano, Nuovo Cim. A 57, 190 (1968).

[5] S. J. Brodsky, G. F. de Teramond, H. G. Dosch and J. Erlich, Phys. Rep. 584, 1 (2015).

[6] M. Ademollo and E. Del Giudice, Nuovo Cim. A 63, 639 (1969).

[7] P. V. Landshoff and J. C. Polkinghorne, Nucl. Phys. B 19, 432 (1970).

[8] A. Deur, S. J. Brodsky and G. F. de Téramond, J. Phys. G 44, no. 10, 105005 (2017).

[9] G. R. Farrar and D. R. Jackson, Phys. Rev. Lett. 35, 1416 (1975).

[10] S. J. Brodsky, M. Burkardt and I. Schmidt, Nucl. Phys. B 441, 197 (1995).

[11] H. Avakian, S. J. Brodsky, A. Deur and F. Yuan, Phys. Rev. Lett. 99, 082001 (2007).

[12] JLab experiment E12-06-110, spokespersons: X. Zheng (contact), G. Cates, J.-P. Chen, and Z.-E. Meziani.

[13] JLab experiment E12-06-122, spokespersons: B. Wojtsekhowski (contact), J. Annand, T. Averett, G. Cates, N. Liyanage, G. Rosner, and X. Zheng. 